\title{
In vivo tracking of platelets: Circulating degranulated platelets rapidly lose surface $P$-selectin but continue to circulate and function
}

\author{
(CD62P/GMP-140/PADGEM protein/flow cytometry/thrombin)
}

\author{
Alan D. Michelson*†, Marc R. Barnard*, Herbert B. Hechtman $\ddagger$, Hollace MacGregor $\S$, \\ RAYMOND J. CONNOLLY $"$, JOSEPH LOSCALZOIl, AND C. ROBERT VALERI§
}

${ }^{*}$ Center for Platelet Function Studies, Departments of Pediatrics and Surgery, University of Massachusetts Medical School, Worcester, MA 01655; $¥$ Department of Surgery, Brigham and Women's Hospital, Harvard Medical School, Boston, MA 02115; \$Naval Blood Research Laboratory and IWhitaker Cardiovascular Institute and Department of Medicine, Boston University School of Medicine, Boston, MA 02118; and "Department of Surgery, New England Medical Center, Tufts University School of Medicine, Boston, MA 02111

Communicated by Richard O. Hynes, Massachusetts Institute of Technology, Cambridge, MA, July 24, 1996 (received for review April 29, 1996)

\begin{abstract}
To examine the hypothesis that surface $P$ selectin-positive (degranulated) platelets are rapidly cleared from the circulation, we developed novel methods for tracking of platelets and measurement of platelet function in vivo. Washed platelets prepared from nonhuman primates (baboons) were labeled with PKH2 (a lipophilic fluorescent dye), thrombin-activated, washed, and reinfused into the same baboons. Three-color whole blood flow cytometry was used to simultaneously (i) identify platelets with a mAb directed against glycoprotein (GP)IIb-IIIa (integrin $\alpha_{1 \mathrm{Ib}} \beta_{3}$ ), (ii) distinguish infused platelets by their PKH2 fluorescence, and (iii) analyze platelet function with mAbs. Two hours after infusion of autologous thrombin-activated platelets (Pselectin-positive, PKH2-labeled), $95 \pm 1 \%$ (mean \pm SEM, $n=$ 5) of the circulating PKH2-labeled platelets had become P-selectin-negative. Compared with platelets not activated with thrombin preinfusion, the recovery of these circulating PKH2-labeled, P-selectin-negative platelets was similar 24 h after infusion and only slightly less $48 \mathrm{~h}$ after infusion. The loss of platelet surface $P$-selectin was fully accounted for by a $67.1 \pm 16.7 \mathrm{ng} / \mathrm{ml}$ increase in the plasma concentration of soluble P-selectin. The circulating PKH2-labeled, P-selectinnegative platelets were still able to function in vivo, as determined by their $(i)$ participation in platelet aggregates emerging from a bleeding time wound, (ii) binding to Dacron in an arteriovenous shunt, (iii) binding of mAb PAC1 (directed against the fibrinogen binding site on GPIIb-IIIa), and (iv) generation of procoagulant platelet-derived microparticles. In summary, $(i)$ circulating degranulated platelets rapidly lose surface $\mathbf{P}$-selectin to the plasma pool, but continue to circulate and function; and (ii) we have developed novel three-color whole blood flow cytometric methods for tracking of platelets and measurement of platelet function in vivo.
\end{abstract}

P-selectin, a member of the selectin family which includes Eand L-selectin, is a cell-adhesion molecule of activated platelets and endothelial cells $(1,2)$. P-selectin (also known as CD62P, GMP-140, and PADGEM protein) is a component of the $\alpha$ granule membrane of resting platelets that is only expressed on the platelet surface membrane during and after platelet degranulation and secretion $(1,2)$. A soluble form of P-selectin circulates in plasma (3).

Platelet surface P-selectin mediates the adherence of degranulated platelets to leukocytes in vitro $(4,5)$ and in vivo $(6)$. It has therefore been postulated that surface P-selectin-positive (degranulated) platelets may be rapidly cleared from the circulation

The publication costs of this article were defrayed in part by page charge payment. This article must therefore be hereby marked "advertisement" in accordance with 18 U.S.C. $\$ 1734$ solely to indicate this fact. by leukocytes $(2,4,5,7,8)$. In apparent contradiction to this postulate, other investigators have reported that degranulated platelets are no more rapidly cleared from the circulation than control platelets $(9,10)$. Methods have not previously been available to directly address this issue.

In this study, we used novel three-color whole blood flow cytometric methods for tracking of circulating degranulated platelets and measurement of their function in vivo. We thereby demonstrate that circulating degranulated platelets rapidly lose surface $\mathrm{P}$-selectin to the plasma pool but continue to circulate and function in vivo. Thus, our studies reconcile the results of previous studies $(2,4,5,7-10)$ by demonstrating that platelet surface P-selectin molecules, rather than degranulated platelets, are rapidly cleared.

\section{METHODS}

Baboons. Studies were performed in baboons (adult male Papio anubis) because these primates are hemostatically similar to man (11). The animals were housed at and cared for according to the standard protocols of the Animal Care Facility of the New England Medical Center, Boston. The protocol for this study was approved by the Institutional Animal Care and Use Committee of Tufts University School of Medicine. Prior to all procedures, the animals were sedated with $250 \mathrm{mg}$ ketamine hydrochloride i.m. and anesthetized with 50-75 mg sodium pentobarbital i.v.

Three-Color Whole Blood Flow Cytometric Analysis of Infused (PKH2-Labeled) Platelets and Noninfused (Non-PKH2Labeled) Platelets in the Same Sample. $\mathrm{PKH} 2$ labeling. $\mathrm{PKH} 2$ (Zynaxis, Malvern, PA), a nonradioactive lipophilic fluorescent dye that binds to the lipid bilayer of the plasma membrane (12), was used as a platelet label. Baboon platelet concentrates were prepared and stored at $22^{\circ} \mathrm{C}$ for not more than $18 \mathrm{~h}$ under standard blood bank conditions (13). Washed platelets were then prepared as described (14), resuspended in Diluent A (Zynaxis) (platelet concentration, $800,000 / \mu \mathrm{l})$, and incubated $\left(22^{\circ} \mathrm{C}, 10\right.$ min) with PKH2 $2 \mu \mathrm{M}$ and prostaglandin (PG) $\mathrm{I}_{2} 2.5 \mu \mathrm{g} / \mathrm{ml}$ (final concentrations). After addition of citrate-albumin buffer (final citrate concentration, $7 \mathrm{mM}$ ) (15), $\mathrm{pH} 6.5$ with $\mathrm{PGE}_{1} 120 \mathrm{ng} / \mathrm{ml}$ (final concentration), samples were centrifuged $(1,250 \times \mathrm{g}, 10$ min, $22^{\circ} \mathrm{C}$ ) and either (i) resuspended in Plasma-Lyte, $\mathrm{pH} 7.4$ (Baxter Health Care, Deerfield, IL) or (ii) resuspended in modified Tyrode's buffer (14), $\mathrm{pH} 7.4$ (platelet concentration, $100,000 / \mu \mathrm{l})$, incubated $\left(22^{\circ} \mathrm{C}, 10 \mathrm{~min}\right)$ with thrombin at $1 \mathrm{unit} / \mathrm{ml}$ (provided by John W. Fenton II, New York Department of Health, Albany), washed with citrate-albumin buffer (final citrate concentration, $8.7 \mathrm{mM}$ ), final $\mathrm{pH} 6.5$ with $\mathrm{PGE}_{1} 120 \mathrm{ng} / \mathrm{ml}$ (final concentration), and resuspended in Plasma-Lyte. Neither the

Abbreviations: GP, glycoprotein; PG, prostaglandin.

†To whom reprint requests should be addressed. 
modified Tyrode's buffer nor the Plasma-Lyte contained any $\mathrm{PGE}_{1}$ or citrate. As determined by forward light scatter, the platelets were not aggregated. As previously reported $(9,10)$, the thrombin-activated platelets could be reproducibly washed and resuspended without difficulty by the use of the following conditions: the presence of $\mathrm{PGE}_{1}$, low $\mathrm{pH}$, high citrate concentration, low platelet concentration, and a pause of $15 \mathrm{~min}$ after the resuspension of thrombin-activated platelets in citrate-albumin buffer.

Expression of P-selectin and the glycoprotein (GP)IIb-IIIa complex (integrin $\alpha_{I I b} \beta_{3}$ ) on the surface of platelets in peripheral blood. Following reinfusion of autologous $\mathrm{PKH} 2$-labeled platelets into a baboon, peripheral blood was drawn into a Vacutainer (Becton Dickinson) at the indicated times (after discarding the first $2 \mathrm{ml}$ of blood). The platelets were analyzed by a modified version of our previously described whole blood cytometric method (16). Three-color whole blood flow cytometry was used to simultaneously $(i)$ identify platelets in whole blood by $(a)$ gating on their characteristic forward and orthogonal light scatter and $(b)$ discriminating on the binding of the R-phycoerythrin-conjugated GPIIb-IIIa-specific mAb 7E3 (17) (provided by Barry S. Coller, Mount Sinai Medical Center, New York); (ii) distinguish the infused platelets by their PKH2 fluorescence; and (iii) determine the platelet surface expression of P-selectin on individual platelets by the binding of biotinylated mAbs S12 or G1 (5) (Centocor, Malvern, PA), followed by streptavidin-RED613 (GIBCO). In some experiments, the platelet surface exposure of fibrinogen binding sites on the GPIIb-IIIa complex was determined by the binding of biotinylated mAb PAC1 (18) (Cell Center, University of Pennsylvania, Philadelphia), followed by streptavidinRED613. PAC1 was added before, and S12 or G1 was added after, fixation with $1 \%$ formaldehyde. For assays with PAC1, antibody 7E3 was added after the PAC1. Background binding, obtained from parallel samples incubated with biotinylated mouse IgG (for assays with S12 or G1) or IgM (for assays with PAC1) (Boehringer Mannheim), was subtracted from each test sample. As indicated in the text, some samples were incubated with platelet agonists prior to antibody incubation and fixation. All samples were analyzed in an EPICS Profile flow cytometer (Coulter) equipped with an argon laser. The fluorescence of PKH2, phycoerythrin, and RED613 were detected by using 525 $\mathrm{nm}, 575 \mathrm{~nm}$, and $635 \mathrm{~nm}$ band pass filters, respectively, with appropriate color compensation. As indicated in Results, some samples were analyzed by the percentage of P-selectin-positive platelets. The percentage of P-selectin-positive platelets was defined as the percentage of platelets that had an S12 fluorescence greater than $98 \%$ of the platelets in matched samples labeled with mouse IgG. For each test mAb [S12, G1, PAC1, and V237 (see below)], an excess of unlabeled antibody inhibited the binding to platelets of labeled antibody.

Generation of procoagulant microparticles in peripheral blood. Three-color whole blood flow cytometry was also used to identify procoagulant platelet-derived microparticles in whole blood by $(i)$ gating on log forward light scatter, (ii) discriminating on the binding of the phycoerythrin-conjugated GPIIb-IIIa-specific $\mathrm{mAb} 7 \mathrm{E} 3$, and (iii) binding of biotinylated mAb V237 (19) (directed against activated coagulation factor $\mathrm{V}$ ) (provided by Charles T. Esmon, Oklahoma Medical Research Foundation, Oklahoma City), as detected by streptavidin-RED613. Background binding, obtained from parallel samples incubated with biotinylated mouse IgG rather than V237, was subtracted from each test sample. Samples were diluted 6-fold in autologous plasma, as a source of additional coagulation factor V. As indicated in the text, some samples were incubated with platelet agonists. Microparticles derived from infused platelets were identified by their PKH2 fluorescence. The number of procoagulant platelet-derived microparticles was expressed as a percentage of the total number of platelets and procoagulant platelet-derived microparticles.
Platelet aggregation in shed blood. Twenty-four hours after the infusion of autologous PKH2-labeled platelets, a standardized bleeding time was performed with a Simplate II device (General Diagnostics, Durham, NC). The emerging blood was immediately fixed in formaldehyde $1 \%$ (final concentration), and platelet aggregation was determined by whole blood flow cytometry as described (20). Platelets were identified in whole blood by binding of the phycoerythrin-conjugated GPIIb-IIIaspecific $\mathrm{mAb}$ 7E3. The noninfused platelets were identified by their lack of $\mathrm{PKH} 2$ fluorescence. The infused platelets in the same blood sample were identified by their PKH2 fluorescence.

Surface P-selectin on platelets adherent to Dacron in an ex vivo shunt. Twenty-four hours after the infusion of autologous PKH2-labeled platelets, peripheral blood was routed through a $6 \mathrm{~mm}$ (diameter) $\times 6 \mathrm{~cm}$ (length) knitted double velour Dacron graft impregnated with purified collagen (Meadox Medicals, Oakland, CA) secured into an ex vivo femoral arteriovenous shunt, as described (21). The Dacron graft is known to be thrombogenic in this model (21). The arteriovenous shunt was clamped after $2 \mathrm{~h}$ of flow and an approximately $4 \times 4 \mathrm{~mm}$ sample of the Dacron graft was immediately excised, placed in modified Hepes-Tyrode's buffer ( $\mathrm{pH} 7.4$ ), mixed gently for $30 \mathrm{~min}$ at $37^{\circ} \mathrm{C}$ with plasmin $1 \mathrm{CU} / \mathrm{ml}$ (Kabi-Pharmacia Hesper, Franklin, $\mathrm{OH}$ ), and fixed in formaldehyde $1 \%$ (final concentration). This concentration of plasmin lysed the fibrin clot and released platelets and plateletderived microparticles without causing platelet degranulation, as determined by lack of platelet surface expression of $\mathrm{P}$ selectin in peripheral blood samples treated identically (data not shown; see also ref. 14). As described above, the released platelets were then analyzed by three-color flow cytometry to determine whether they were $\mathrm{PKH} 2$-positive or -negative and to determine their surface expression of P-selectin.

Percent Recovery of Infused (PKH2-Labeled) Platelets. At the indicated times after the infusion of autologous PKH2labeled platelets, peripheral blood was drawn into a Vacutainer, as described above. Percent recovery of infused (PKH2labeled) platelets at each time point was determined by:

\section{(Percent PKH2-positive platelets $) \times($ platelet count $/ \mu \mathrm{l})$} $\times$ [blood volume $(\mu \mathrm{l})]$

(number of PKH2-positive platelets infused)

Blood volume was calculated from the plasma volume [determined by the ${ }^{125}$ I-labeled $\left({ }^{125} \mathrm{I}\right)$ albumin method] and the total body hematocrit (peripheral venous hematocrit $\times 0.87$ ), as described (22). Platelet counts were determined in a Coulter. JT analyzer.

Plasma P-Selectin Assay. Peripheral blood was drawn into a Vacutainer at the indicated times postinfusion, as described above, and centrifuged $\left(1500 \times \mathrm{g}, 20 \mathrm{~min}, 4^{\circ} \mathrm{C}\right)$. The plasma was separated and stored at $-20^{\circ} \mathrm{C}$. After thawing, the plasma was centrifuged $(10,000 \times g, 10 \mathrm{~min})$ before being assayed. In some assays (as indicated), the plasma was subjected to an additional centrifugation $(100,000 \times g, 2 \mathrm{~h})$ to remove any platelet-derived microparticles (23). Plasma P-selectin was determined by a triple layer ELISA (24). Microtiter plates were sequentially incubated with $\mathrm{S} 12,5 \%$ nonfat dry milk, purified soluble P-selectin (Centocor) or test plasma, a chimeric version of the murine anti-human P-selectin mAb G1 (Centocor) (human $F_{c}$ region), alkaline phosphataseconjugated rabbit anti-human IgG $\left(F_{c}\right)$ (Accurate Chemicals), alkaline phosphatase substrate $(1 \mathrm{mg}$ P-Nitro phenyl phosphate per $1 \mathrm{ml} 0.1 \mathrm{M}$ diethanolamine) (Sigma), and $3 \mathrm{M}$ $\mathrm{NaOH}$; they were then read for absorbance at $405 \mathrm{~nm}$. 


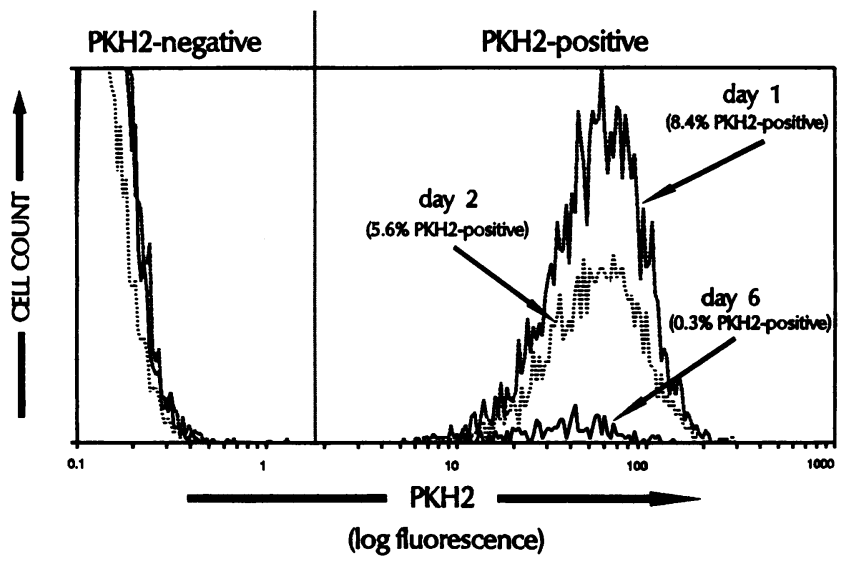

FIG. 1. Ability of whole blood flow cytometry to distinguish infused, PKH2-labeled platelets in vivo. Autologous washed thrombinactivated PKH2-labeled platelets were transfused into a baboon. Peripheral blood was drawn from the baboon on days 1,2 , and 6 . Platelets were identified in whole blood by light scatter and binding of the GPIIb-IIIa-specific mAb 7E3. The proportion of all circulating platelets that are $\mathrm{PKH} 2$-positive are indicated. The experiment is representative of 10 experiments so performed.

\section{RESULTS}

In Vivo Identification of Infused Platelets. Infused platelets were easily discriminated from noninfused platelets by whole blood flow cytometry because PKH2-labeled platelets were $\approx 300$-fold more fluorescent than unlabeled platelets (Fig. 1). A subpopulation of $\mathrm{PKH} 2$-labeled platelets as small as $0.3 \%$ of all platelets could be identified in vivo (Fig. 1). PKH2 binding to platelets was stable in vivo, as shown by the fact that PKH2-labeled platelets still circulating on day 6 had $85.0 \pm$ $6.1 \%$ (mean \pm SEM, $n=8$ ) of the fluorescence of the PKH2-labeled platelets circulating on day 1 (Fig. 1).

Circulating Degranulated Platelets Rapidly Lose Surface PSelectin. As demonstrated in Fig. 2, infused thrombin-activated PKH2-labeled platelets initially circulated in a P-selectin-positive form (quadrant 2 of the 3-min time point) but, after a period of sequestration [as previously described (25)] (lower density of dots in quadrants 2 and 4 of the $10-\mathrm{min}$ and 30 -min time points), gradually reappeared as P-selectin-negative platelets (quadrant 4 of the 2-, 3-, and 24-h time points). Two hours after the infusion of the autologous thrombin-activated platelets (P-selectinpositive, $\mathrm{PKH} 2$-labeled), $95 \pm 1 \%(n=5)$ of the circulating PKH2-labeled platelets had become P-selectin-negative (Fig. 2) and there was a $97.0 \pm 0.6 \%(n=5)$ average decrease in the platelet surface expression of P-selectin on the circulating $\mathrm{PKH} 2-$ labeled platelets (Fig. $3 A$ ).
Etiology of the Decreased Platelet Surface Expression of P-Selectin on Circulating Degranulated Platelets. The decreased platelet surface expression of P-selectin on circulating degranulated platelets did not appear to be the result of a conformational change in P-selectin, because parallel results were obtained with two different P-selectin-specific mAbs known to be directed against different epitopes (5): S12 (Figs. 2 and $3 A$ ) and $\mathrm{G} 1$ (data not shown).

The loss of platelet surface P-selectin was associated with a $52.7 \pm 18.8 \%(67.1 \pm 16.7 \mathrm{ng} / \mathrm{ml})$ maximal increase in the plasma concentration of soluble P-selectin (Fig. $3 B$ ), which accounted for $93.5 \pm 23.8 \%$ of the calculated loss of platelet surface P-selectin. This calculation was based on the available platelet surface P-selectin $(77.3 \pm 8.1 \%$ of maximum: Fig. $3 A$, "platelet unit preinfusion" time point), the number of platelets infused $\left(3.5 \pm 0.9 \times 10^{10}\right)$, the plasma volume $[940.6 \pm 113.6$ $\mathrm{ml}$, as determined by the ${ }^{125} \mathrm{I}$-albumin method (22)], the molecular weight of plasma P-selectin (137 kDa) (3), and the estimated number of copies of $P$-selectin per platelet $(13,000)$ (2). At all measured times, plasma P-selectin was not reduced by centrifugation of the plasma at $100,000 \times g$ for $2 \mathrm{~h}$, thereby demonstrating that the assay was not measuring P-selectin in platelet-derived microparticles (23). In control experiments in which platelets were not thrombin-activated preinfusion, the circulating $\mathrm{PKH} 2$-labeled platelets were $\mathrm{P}$-selectin-negative at all times (Fig. $3 C$ ) (demonstrating that $\mathrm{PKH} 2$ labeling of platelets did not result in significant degranulation) and there was no increase in plasma P-selectin (Fig. 3D).

Despite Their Loss of Surface P-Selectin, Degranulated Platelets Are Not Rapidly Removed from the Circulation. Platelets that were thrombin-activated preinfusion sequestrated (25) earlier and longer than platelets not thrombinactivated preinfusion (Fig. 4). However, $24 \mathrm{~h}$ after infusion, the recovery of platelets thrombin-activated preinfusion was similar to the recovery of platelets not thrombin-activated preinfusion, and was only slightly less $48 \mathrm{~h}$ after infusion (Fig. 4).

Despite Their Loss of Surface P-Selectin, Circulating Degranulated Platelets Continue to Function. Despite their degranulation and subsequent loss of surface P-selectin, circulating PKH2-labeled platelets that had been thrombinactivated preinfusion were still able to function $24 \mathrm{~h}$ after infusion, as determined by four independent methods.

(i) Platelets thrombin-activated preinfusion were demonstrated to function in vivo $24 \mathrm{~h}$ after their infusion, as determined by their presence (dots in Fig. $5 B$ ) in platelet aggregates in the shed blood emerging from a standardized bleeding time wound. As determined by percent positive analysis on the log forward light scatter signal [a measure of percent aggregate formation (20)], PKH2-positive platelets formed aggregates in shed blood to at least the same extent as PKH2-negative platelets in the same
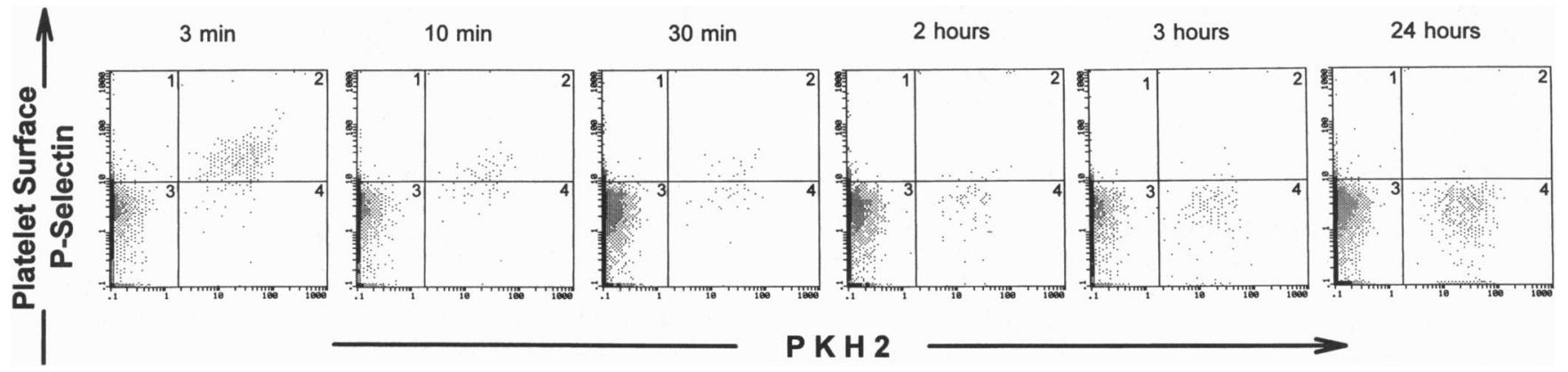

Fig. 2. Platelet surface expression of P-selectin on degranulated platelets infused in vivo, as determined by three-color whole blood flow cytometry. Autologous washed thrombin-activated PKH2-labeled platelets were transfused into a baboon. Peripheral blood was drawn from the baboon at the indicated times and immediately fixed with formaldehyde $1 \%$ (final concentration). Platelets were identified in whole blood by light scatter and binding of the phycoerythrin-conjugated GPIIb-IIIa-specific mAb 7E3. The platelet surface expression of P-selectin was determined by the binding of biotinylated mAb S12 followed by streptavidin-RED613 (vertical axis). On each individual platelet, PKH2 fluorescence was measured simultaneously (horizontal axis). The experiment is representative of six experiments so performed. 

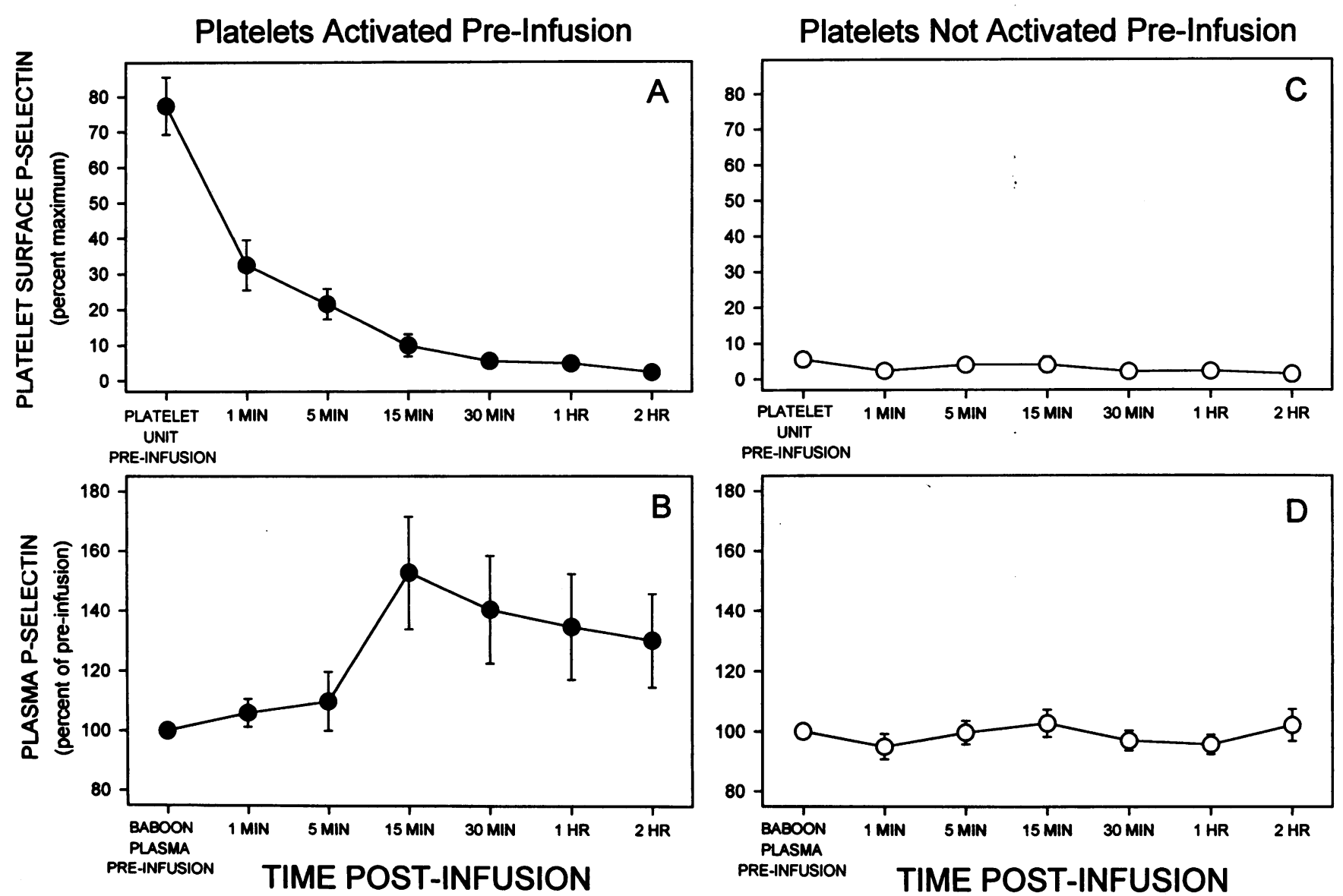

Fig. 3. Platelet surface P-selectin $(A$ and $C)$ and plasma $P$-selectin $(B$ and $D)$ in peripheral blood samples from experiments in which platelets were thrombin-activated preinfusion $(A$ and $B)$ or not thrombin-activated preinfusion $(C$ and $D)$. Autologous washed PKH2-labeled platelets were transfused into baboons. Peripheral blood, drawn from the baboons at the times indicated on the horizontal axis, was immediately either formaldehyde fixed for flow cytometric analysis or centrifuged to separate the plasma. $(A$ and $C)$ Three-color whole blood flow cytometry. Platelets were identified in whole blood by light scatter and binding of the phycoerythrin-conjugated GPIIb-IIIa-specific mAb 7E3. The infused platelets were identified by their PKH2 fluorescence. The platelet surface expression of P-selectin (vertical axis) was determined by the binding of the biotinylated mAb S12, followed by streptavidin-RED613. Maximum platelet surface P-selectin was defined as the binding of S12 to platelets in preinfusion peripheral blood samples after stimulation at $22^{\circ} \mathrm{C}$ for $15 \mathrm{~min}$ with thrombin $10 \mathrm{units} / \mathrm{ml}$. $(B$ and $D)$ Plasma P-selectin was determined by ELISA. Data are mean \pm SEM, $n=5$.

sample, irrespective of whether the platelets were or were not thrombin-activated preinfusion $(n=6)$.

(ii) As determined by platelet surface exposure of the fibrinogen binding site on the GPIIb-IIIa complex [reported by mAb PAC1 (18)], platelets thrombin-activated preinfusion were able to function normally in $24-\mathrm{h}$ postinfusion peripheral blood stimulated ex vivo by ADP and epinephrine, although poorly in response to a second challenge with thrombin (Fig. $6 \mathrm{~A}$ ).

(iii) Platelets thrombin-activated preinfusion were able to function as determined by their generation of procoagulant platelet-derived microparticles (19) in response to ex vivo stimulation of peripheral blood drawn $24 \mathrm{~h}$ postinfusion (Fig. 7A).

(iv) Platelets thrombin-activated preinfusion were able to function normally as determined by their adherence to Dacron in an arteriovenous shunt. Three-color flow cytometry was used to calculate the ratio of $\mathrm{PKH} 2$-positive to $\mathrm{PKH} 2$-negative platelets (i) adherent to the Dacron and (ii) in peripheral blood. By these means, we determined that platelets thrombin-activated preinfusion (PKH2-positive) adhered to the Dacron 106.0 $\pm 1.9 \%(n=$ 3 ) as well as the noninfused (PKH2-negative) platelets.

PKH2 Does Not Interfere with Platelet Function. PKH2 did not interfere with platelet function, as demonstrated by the following six independent methods in which infused autologous PKH2-labeled platelets not preincubated with thrombin functioned as well as circulating non-PKH2-labeled platelets simultaneously analyzed in the same sample: $(i)$ participation in platelet aggregates emerging from a bleeding time wound (Fig. 5D); (ii) exposure of fibrinogen binding sites on the GPIIb-IIIa complex in response to ex vivo stimulation of peripheral blood (Fig. 6B); (iii) platelet surface expression of $\mathrm{P}$-selectin in response to ex vivo stimulation of peripheral blood with either thrombin, phorbol myristate acetate (Sigma), or a combination of ADP and epinephrine (data not shown); (iv) generation of procoagulant platelet-derived microparticles in response to ex vivo stimulation of peripheral blood (Fig. 7B); (v) infused ( $\mathrm{PKH} 2$-positive) platelets adhered to Dacron in the arteriovenous shunt $112.3 \pm 5.1 \%(n=3)$ as well as the noninfused (PKH2-negative) platelets; and (vi) expression of surface P-selectin in response to adherence to Dacron in the ex vivo shunt (data not shown).

\section{DISCUSSION}

In this study, we demonstrate that circulating degranulated platelets rapidly lose surface P-selectin to the plasma pool, but continue to circulate. Furthermore, we show that, despite their loss of surface P-selectin, circulating degranulated platelets continue to function in vivo as determined by four independent methods: participation in platelet aggregation, exposure of the fibrinogen binding site on the GPIIb-IIIa complex, adherence to Dacron in an arteriovenous shunt, and generation of procoagulant platelet-derived microparticles. 


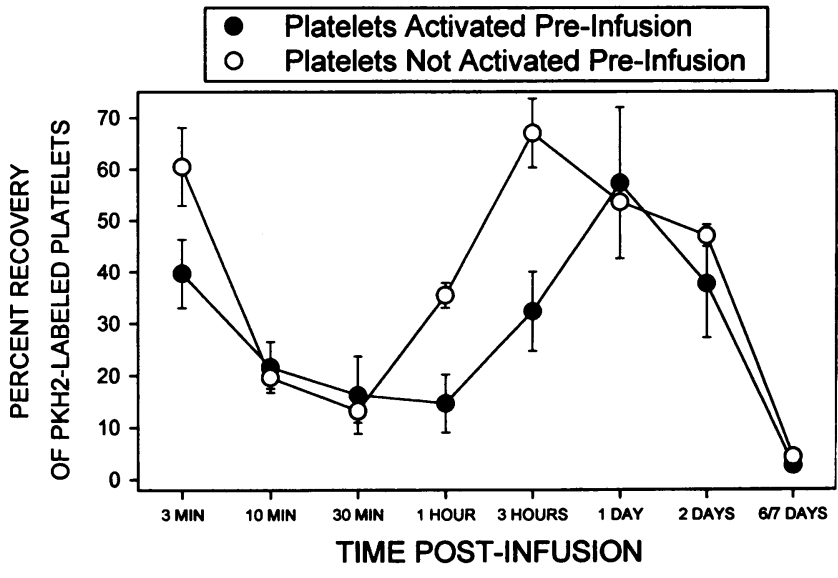

FIG. 4. Recovery of platelets thrombin-activated preinfusion compared with platelets not thrombin-activated preinfusion, as determined by whole blood flow cytometry. Autologous washed PKH2labeled platelets were transfused into baboons. Peripheral blood was drawn from the baboons at the times indicated on the horizontal axis and immediately fixed with formaldehyde. Platelets were identified in whole blood by light scatter and binding of the phycoerythrinconjugated GPIIb-IIIa-specific mAb 7E3. The infused platelets were identified by their PKH2 fluorescence. Percent recovery of PKH2labeled platelets (vertical axis) was determined as described. Data are mean \pm SEM, $n=5$.

Thrombus formation is associated with local generation of agonists-e.g., thrombin, that induce platelet degranulation. De-

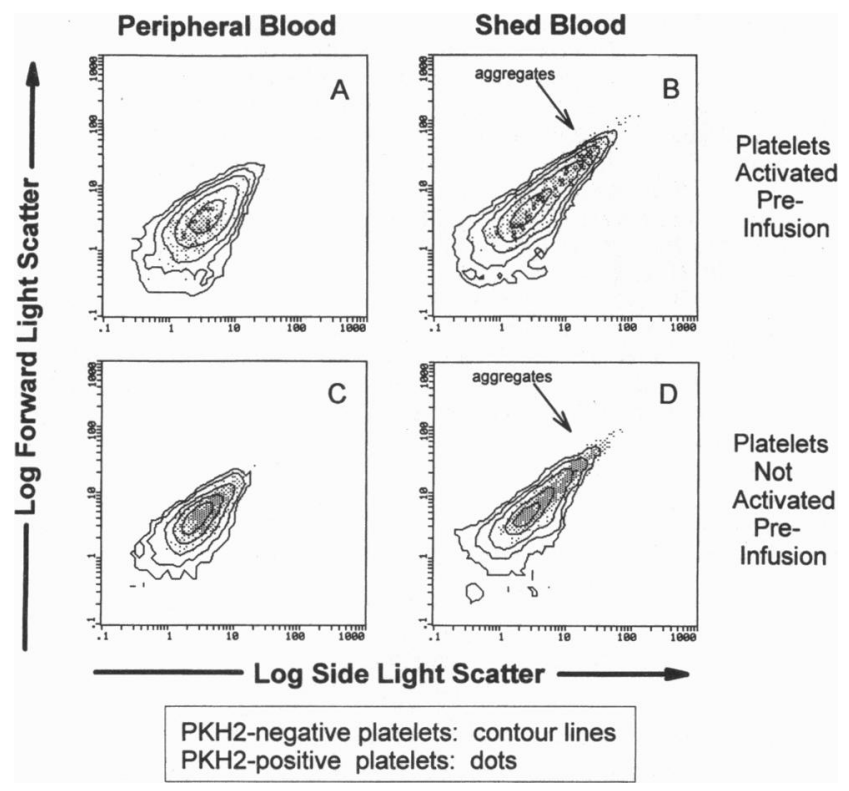

Fig. 5. In vivo function of platelets thrombin-activated preinfusion $(A$ and $B)$ or not thrombin-activated preinfusion $(C$ and $D)$, as determined by their presence in platelet aggregates emerging from a standardized bleeding time wound. Twenty-four hours after infusion of autologous PKH2-labeled platelets, peripheral blood $(A$ and $C$ ) and the blood emerging from the wound 2 min after the bleeding time incision (shed blood, $B$ and $D$ ) was drawn, immediately fixed with formaldehyde, and analyzed for platelet aggregation by whole blood flow cytometry. Platelets were identified in whole blood by light scatter and binding of the phycoerythrin-conjugated GPIIb-IIIa-specific $\mathrm{mAb}$ 7E3. The infused platelets (dots) were identified by their PKH2 fluorescence. The noninfused platelets in the same blood sample (contour lines) were identified by their lack of $\mathrm{PKH} 2$ fluorescence. (The different density of dots in $A$ and $B$ compared with $C$ and $D$ resulted from infusion of different numbers of $\mathrm{PKH} 2$-positive platelets in these different experiments.) The experiment is representative of six experiments so performed.

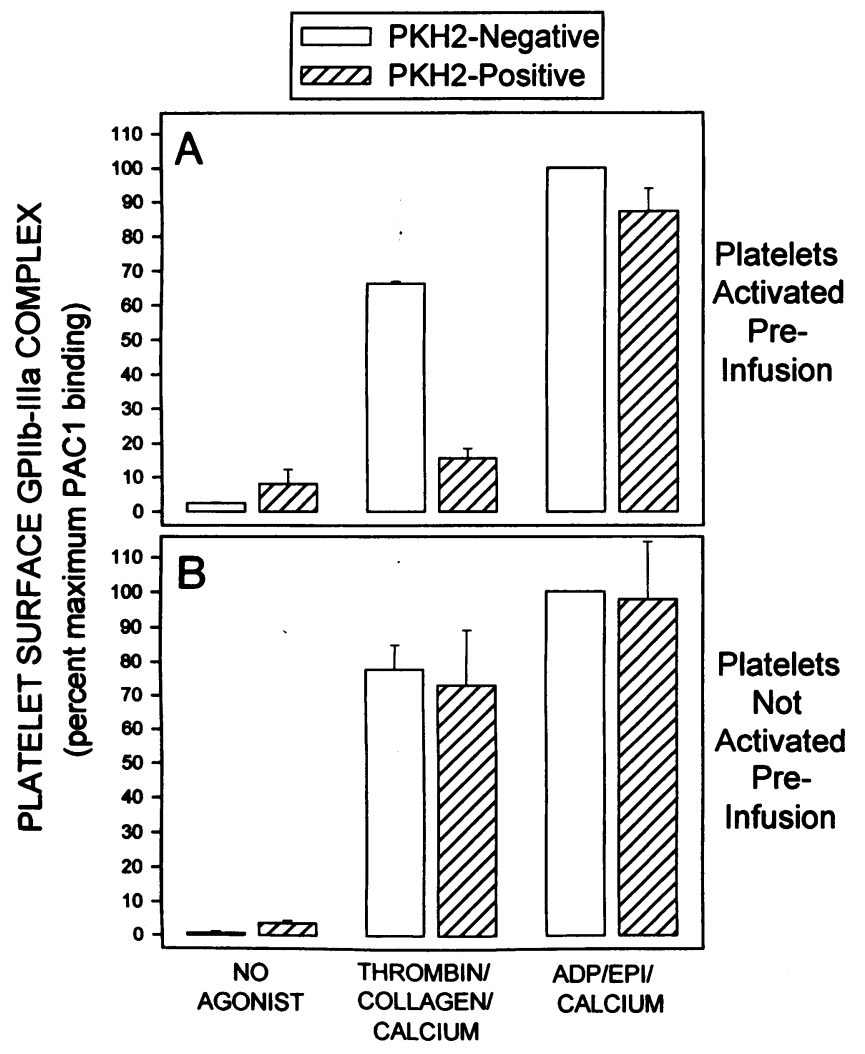

Fig. 6. Ex vivo function of platelets thrombin-activated preinfusion $(A)$ or not thrombin-activated preinfusion $(B)$, as determined by their exposure of the fibrinogen binding site on the GPIIb-IIIa complex. Autologous washed PKH2-labeled platelets were transfused into baboons. Peripheral blood drawn from the baboons $24 \mathrm{~h}$ postinfusion was incubated $\left(37^{\circ} \mathrm{C}, 10 \mathrm{~min}\right)$ with $2 \mathrm{mM}$ Gly-Pro-Arg-Pro (Calbiochem) [an inhibitor of fibrin polymerization (16)] and either $(i)$ thrombin 10 units $/ \mathrm{ml}$, collagen type I $20 \mu \mathrm{g} / \mathrm{ml}$ (Hormon-Chemie, Munich), and $3 \mathrm{mM} \mathrm{CaCl} 2$; (ii) $20 \mu \mathrm{M}$ ADP (BioData, Horsham, PA), $20 \mu \mathrm{M}$ epinephrine (BioData), and $3 \mathrm{mM} \mathrm{CaCl}_{2}$; or (iii) buffer only. The samples were then fixed with formaldehyde and analyzed by three-color whole blood flow cytometry. Platelets were identified in whole blood by light scatter and binding of the phycoerythrinconjugated GPIIb-IIIa-specific mAb 7E3. The infused platelets were identified by their $\mathrm{PKH} 2$ fluorescence. The platelet surface exposure of fibrinogen binding sites on the GPIIb-IIIa complex (vertical axis) was determined by the binding of the biotinylated mAb PAC1 (added prior to 7E3), followed by streptavidin-RED613. PAC1 binding to PKH2-negative platelets after stimulation $\left(37^{\circ} \mathrm{C}, 10 \mathrm{~min}\right)$ with $20 \mu \mathrm{M}$ $\mathrm{ADP}, 20 \mu \mathrm{M}$ epinephrine, and $3 \mathrm{mM} \mathrm{CaCl}_{2}$ was defined as $100 \%$. Data are mean \pm SEM, $n=3$ in $A$ and $n=5$ in $B$.

granulated platelets that fail to localize to the thrombus, because of the continuing flow of blood, have been hypothesized to be rapidly cleared from the circulation by leukocytes via a platelet surface P-selectin-dependent mechanism $(2,4,5)$. However, it has not been possible to differentiate between clearance of $\mathrm{P}$-selectin-positive platelets and a change in the platelet surface expression of P-selectin. The present study demonstrates in vivo that circulating degranulated platelets rapidly lose surface Pselectin to the plasma pool, thereby enabling the continued circulation and function of degranulated platelets that fail to localize to the thrombus. Thus, our results provide a possible mechanism for the preservation of functional platelets.

No difference in platelet clearance would be expected between thrombin activation in vivo and, as performed in this study, thrombin activation of washed platelets in vitro. Although the platelets in this study were activated after washing away plasma proteins (which could influence platelet clearance), plasma adhesive glycoproteins (fibrinogen, von Wille- 


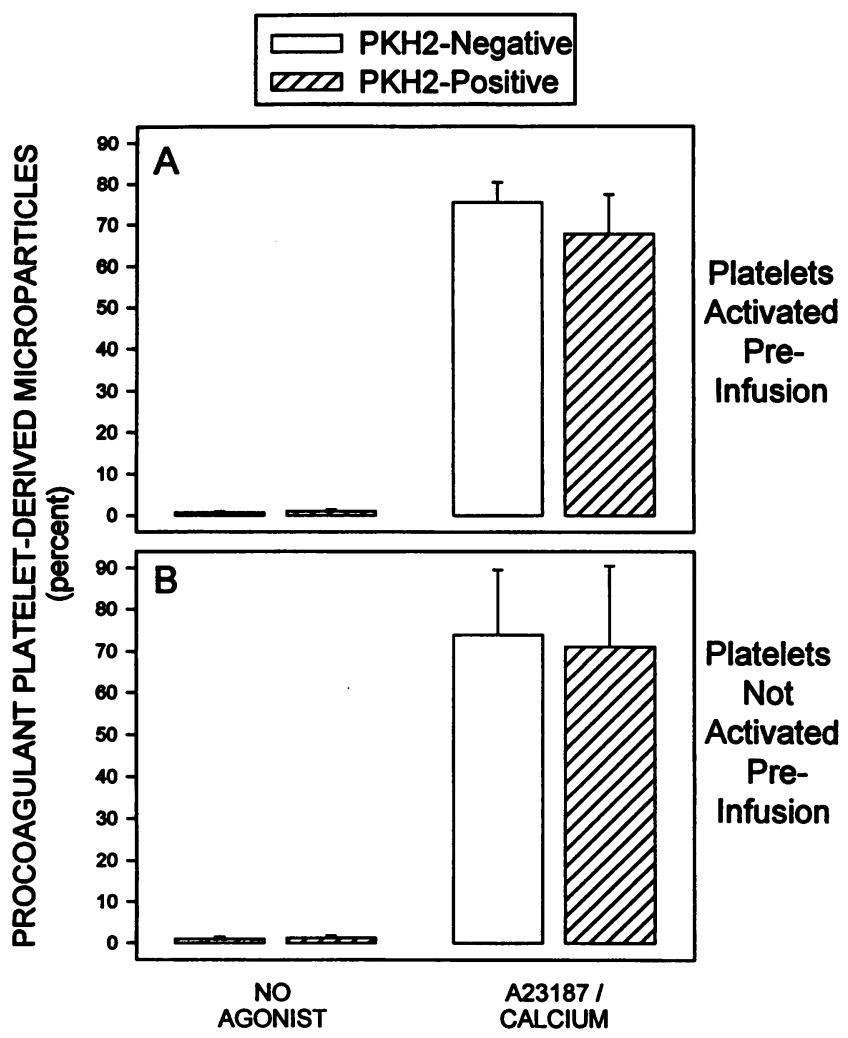

FIG. 7. Ex vivo function of platelets thrombin-activated preinfusion $(A)$ or not thrombin-activated preinfusion $(B)$, as determined by their generation of procoagulant platelet-derived microparticles. Autologous washed PKH2-labeled platelets were transfused into baboons. Peripheral blood drawn from the baboons $24 \mathrm{~h}$ postinfusion was incubated $\left(37^{\circ} \mathrm{C}, 10\right.$ $\mathrm{min}$ ) with $2 \mathrm{mM}$ Gly-Pro-Arg-Pro and either (i) the calcium ionophore A23187 (20 $\mu \mathrm{M}$; Sigma) and $3 \mathrm{mM} \mathrm{CaCl}_{2}$ or (ii) buffer only, and then fixed with formaldehyde. Three-color flow cytometry was used to identify procoagulant platelet-derived microparticles in whole blood by $(i)$ log forward light scatter, (ii) binding of a phycoerythrin-conjugated GPIIbIIIa-specific mAb 7E3, and (iii) binding of a biotinylated mAb (V237) directed against coagulation factor $\mathrm{V}$ (followed by streptavidinRED613). Microparticles derived from the infused platelets were identified by their PKH 2 fluorescence. The number of procoagulant plateletderived microparticles was expressed as a percentage of the total number of platelets and procoagulant platelet-derived microparticles. Data are mean $\pm \operatorname{SEM}, n=4$ in $A$ and $n=3$ in $B$.

brand factor, thrombospondin, fibronectin, vitronectin) are also present in platelet $\alpha$ granules. Thrombin-induced degranulation resulted in release of these adhesive glycoproteins from the $\alpha$ granules, making them available for binding to the platelet surface. Furthermore, immediately upon infusion the degranulated platelets were exposed to plasma proteins.

Although degranulated platelets that had shed surface $P$-selectin performed normally in a number of assays of platelet function, our studies should not be taken to imply that these platelets have completely normal function or that surface $P$-selectin plays no role in platelet function. It has previously been established in vivo that the degranulated platelets present in local thrombi promote leukocyte accumulation via a platelet surface P-selectin-dependent mechanism (6). The role of platelet surface P-selectin in the homing of leukocytes to sites of inflammation could be further studied in our model. Platelet surface P-selectin has also been shown to induce the expression of tissue factor on monocytes (26).

There has been considerable interest in the potential use of platelet surface P-selectin as a marker for the detection of circulating degranulated platelets in clinical settings (e.g., acute coronary artery syndromes, transfusion of platelet concentrates)
(27). However, the present demonstration that circulating degranulated platelets rapidly lose surface P-selectin to the plasma pool indicates that platelet surface P-selectin is not an ideal marker for the detection of circulating degranulated platelets. Nevertheless, platelet surface P-selectin may still be a useful marker of platelet degranulation if $(i)$ the blood sample is drawn immediately distal to the site of platelet activation, (ii) the blood sample is drawn within 5 min of the activating stimulus, or (iii) there is continuous activation of platelets. The present study and previous studies (28) suggest that assay of the plasma concentration of soluble P-selectin could be used as a marker of platelet activation in clinical settings. However, an increase in the plasma concentration of soluble P-selectin in clinical settings may also reflect the release of P-selectin from activated and/or damaged endothelial cells (28).

Despite recent progress in our understanding platelet function in vitro, there remains a paucity of methods to study platelet function in vivo. In this study, we used novel three color whole blood flow cytometric methods for in vivo tracking of infused platelets and measurement of their function. These methods should provide the means to answer many previously difficult to address questions about in vivo platelet function.

This work was supported by National Institutes of Health Grants GM35141-09, HL47416, HL48743, and P50 HL55993, a Veterans Affairs Merit Award, and the U.S. Navy (Office of Naval Research Contract N00014-94-C-0149) with the funds provided by the Naval Medical Research and Development Command.

1. McEver, R. P. (1994) Curr. Opin. Immunol. 6, 75-84.

2. Celi, A., Furie, B. \& Furie, B. C. (1991) Proc. Soc. Exp. Biol. Med. 198, 703-709.

3. Dunlop, L. C., Skinner, M. P., Bendall, L. J., Favaloro, E. J., Castaldi, P. A., Gorman, J. J., Gamble, J. R., Vadas, M. A. \& Berndt, M. C. (1992) J. Exp. Med. 175, 1147-1150.

4. Larsen, E., Celi, A., Gilbert, G. E., Furie, B. C., Erban, J. K., Bonfanti, R., Wagner, D. D. \& Furie, B. (1989) Cell 59, 305-312.

5. Hamburger, S. A. \& McEver, R. P. (1990) Blood 75, 550-554.

6. Palabrica, T., Lobb, R., Furie, B. C., Aronovitz, M., Benjamin, C., Hsu Y. M., Sajer, S. A. \& Furie, B. (1992) Nature (London) 359, 848-851.

7. Rinder, H. M., Murphy, M., Mitchell, J. G., Stocks, J., Ault, K. A. \& Hillman, R. S. (1991) Transfusion 31, 409-414.

8. Triulzi, D. J., Kickler, T. S. \& Braine, H. G. (1992) Transfusion 32, 529-533.

9. Reimers, H. J., Packham, M. A., Kinlough-Rathbone, R. L. \& Mustard J. F. (1973) Br. J. Haematol. 25, 675-689.

10. Reimers, H. J., Kinlough-Rathbone, R. L., Cazenave, J. P., Senyi, A. F., Hirsh, J., Packham, M. A. \& Mustard, J. F. (1976) Thromb. Haemostasis 35, 151-166.

11. Michelson, A. D., Benoit, S. E., Barnard, M. R., MacGregor, H. \& Valeri, C. R. (1995) in Leucocyte Typing V, eds. Schlossman, S. F., Boumsell, L., Gilks, W., Harlan, J. M., Kishimoto, T., Morimoto, C., Ritz, J., Shaw, S., Silverstein, R. L., Springer, T. A., Tedder, T. F. \& Todd, R. F. (Oxford Univ. Press, Oxford), pp. 1230-1231.

12. Horan, P. K. \& Slezak, S. E. (1989) Nature (London) 340, 167-168

13. Valeri, C. R. (1974) N. Engl. J. Med. 290, 353-358.

14. Michelson, A. D. \& Barnard, M. R. (1990) Blood 76, 2005-2010.

15. Yamamoto, N., Greco, N. J., Barnard, M. R., Tanoue, K., Yamazaki, H., Jamieson, G. A. \& Michelson, A. D. (1991) Blood 77, 1740-1748.

16. Michelson, A. D., Ellis, P. A., Barnard, M. R., Matic, G. B., Viles, A. F. \& Kestin, A. S. (1991) Blood 77, 770-779.

17. Coller, B. S. (1985) J. Clin. Invest..76, 101-108.

18. Shattil, S. J., Hoxie, J. A., Cunningham, M. \& Brass, L. F. (1985) J. Biol. Chem. 260, 11107-11114.

19. Sims, P. J., Faioni, E. M., Wiedmer, T. \& Shattil, S. J. (1988) J. Biol. Chem. 263, 18205-18212.

20. Michelson, A. D., MacGregor, H., Barnard, M. R., Kestin, A. S., Rohrer, M. J. \& Valeri, C. R. (1994) Thromb. Haemostasis 71, 633-640.

21. Shoenfeld, N. A., Connolly, R., Ramberg, K., Valeri, C. R., EldrupJorgensen, J. \& Callow, A. D. (1988) Surg. Gynecol. Obstet. 166, 454-457.

22. Valeri, C. R., Lindberg, J. R., Contreras, T. J., Pivacek, L. E., Austin, R. M., Valeri, D. A., Gray, A. \& Emerson, C. P. (1981) Am. J. Vet. Res. 42, 1025-1029.

23. George, J. N., Thoi, L. L., McManus, L. M. \& Reimann, T. A. (1982) Blood 60, 834-840.

24. Harlow, E. \& Lane, D. (1988) Antibodies: A Laboratory Manual (Cold Spring Harbor Lab. Press, Plainview, NY).

25. Franco, R. S., Lee, K. N., Barker-Gear, R., Gates, R. \& Menitove, J. E. (1994) Transfusion 34, 784-789.

26. Celi, A., Pellegrini, G., Lorenzet, R., De Blasi, A., Ready, N., Furie, B. C \& Furie, B. (1994) Proc. Natl. Acad. Sci. USA 91, 8767-8771.

27. Michelson, A. D. (1996) Blood 87, 4925-4936.

28. Chong, B. H., Murray, B., Berndt, M. C., Dunlop, L. C., Brighton, T. \& Chesterman, C. N. (1994) Blood 83, 1535-1541: 